\title{
Exploring and Exploiting the Limited Utility of Captions in Recognizing Intention in Information Graphics*
}

\author{
Stephanie Elzer $^{1}$ and Sandra Carberry ${ }^{2}$ and Daniel Chester ${ }^{2}$ and Seniz Demir ${ }^{2}$ and \\ Nancy Green ${ }^{3}$ and Ingrid Zukerman ${ }^{4}$ and Keith Trnka ${ }^{2}$ \\ ${ }^{1}$ Dept. of Computer Science, Millersville University, Millersville, PA 17551 \\ ${ }^{2}$ Dept. of Computer Science, University of Delaware, Newark, DE 19716 \\ ${ }^{3}$ Dept. of Mathematical Sciences, Univ. of NC at Greensboro, Greensboro, NC 27402 \\ ${ }^{4}$ School of CS \& Software Engrg, Monash Univ., Clayton, Victoria 3800 Australia
}

\begin{abstract}
This paper presents a corpus study that explores the extent to which captions contribute to recognizing the intended message of an information graphic. It then presents an implemented graphic interpretation system that takes into account a variety of communicative signals, and an evaluation study showing that evidence obtained from shallow processing of the graphic's caption has a significant impact on the system's success. This work is part of a larger project whose goal is to provide sight-impaired users with effective access to information graphics.
\end{abstract}

\section{Introduction}

Language research has posited that a speaker or writer executes a speech act whose intended meaning he expects the listener to be able to deduce, and that the listener identifies the intended meaning by reasoning about the observed signals and the mutual beliefs of author and interpreter (Grice, 1969; Clark, 1996). But as noted by Clark (Clark, 1996), language is more than just words. It is any "signal" (or lack of signal when one is expected), where a signal is a deliberate action that is intended to convey a message.

Although some information graphics are only intended to display data values, the overwhelming majority of the graphics that we have examined (taken

\footnotetext{
*Authors can be reached via email as follows: elzer@cs.millersville.edu, nlgreen@uncg.edu, \{carberry, chester, demir, trnka\}@ cis.udel.edu, Ingrid.Zukerman@infotech.monash.edu.au.
}

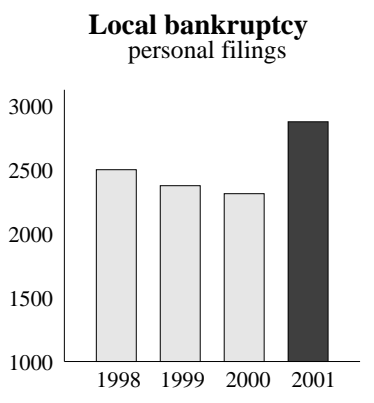

Figure 1: Graphic from a 2001 Local Newspaper

from newspaper, magazine, and web articles) appear to have some underlying goal or intended message, such as the graphic in Figure 1 whose communicative goal is ostensibly to convey the sharp increase in local bankruptcies in the current year compared with the previous decreasing trend. Applying Clark's view of language, it is reasonable to presume that the author of an information graphic expects the viewer to deduce from the graphic the message that the graphic was intended to convey, by reasoning about the graphic itself, the salience of entities in the graphic, and the graphic's caption.

This paper adopts Clark's view of language as any deliberate signal that is intended to convey a message. Section 3 investigates the kinds of signals used in information graphics. Section 4 presents a corpus study that investigates the extent to which captions capture the message of the graphic, illustrates the issues that would arise in trying to fully understand such captions, and proposes shallow processing of the caption to extract evidence from it. Section 5 then describes how evidence obtained from a variety of communicative signals, including shallow processing of the graphic's caption, is used in a probabilistic system for hypothesizing the intended message of the graphic. Section 6 presents an eval- 


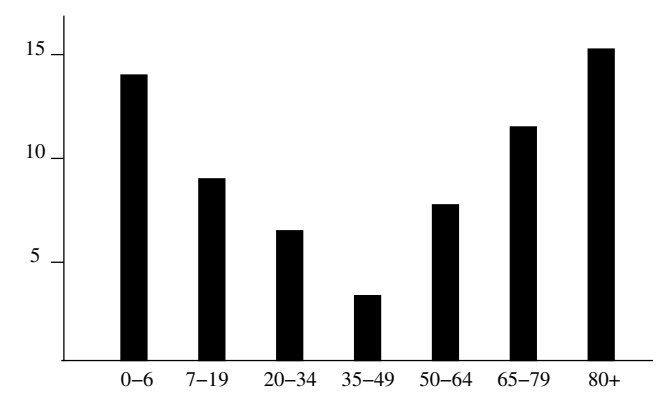

(a)

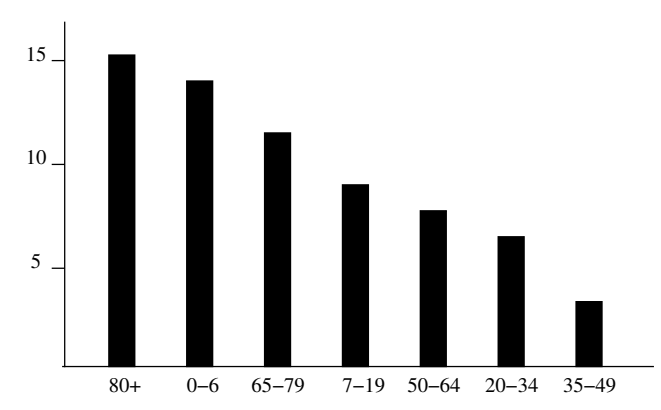

(b)

Figure 2: Two Alternative Graphs from the Same Data

uation showing the system's success, with particular attention given to the impact of evidence from shallow processing of the caption, and Section 7 discusses future work.

Although we believe that our findings are extendible to other kinds of information graphics, our current work focuses on bar charts. This research is part of a larger project whose goal is a natural language system that will provide effective access to information graphics for individuals with sight impairments, by inferring the intended message underlying the graphic, providing an initial summary of the graphic that includes the intended message along with notable features of the graphic, and then responding to follow-up questions from the user.

\section{Related Work}

Our work is related to efforts on graph summarization. (Yu et al., 2002) used pattern recognition techniques to summarize interesting features of automatically generated graphs of time-series data from a gas turbine engine. (Futrelle and Nikolakis, 1995) developed a constraint grammar for parsing vectorbased visual displays and producing representations of the elements comprising the display. The goal of Futrelle's project is to produce a graphic that summarizes one or more graphics from a document (Futrelle, 1999). The summary graphic might be a simplification of a graphic or a merger of several graphics from the document, along with an appropriate summary caption. Thus the end result of summarization will itself be a graphic. The long range goal of our project, on the other hand, is to provide alternative access to information graphics via an initial textual summary followed by an interactive followup component for additional information. The in- tended message of the graphic will be an important component of the initial summary, and hypothesizing it is the goal of our current work.

\section{Evidence about the Intended Message}

The graphic designer has many alternative ways of designing a graphic; different designs contain different communicative signals and thus convey different communicative intents. For example, consider the two graphics in Figure 2. The graphic in Figure 2 a conveys that average doctor visits per year is U-shaped by age; it starts out high when one is very young, decreases into middle age, and then rises again as one ages. The graphic in Figure $2 b$ presents the same data; but instead of conveying a trend, this graphic seems to convey that the elderly and the young have the highest number of doctor visits per year. These graphics illustrate how choice of design affects the message that the graphic conveys.

Following the AutoBrief work (Kerpedjiev and Roth, 2000) (Green et al., 2004) on generating graphics that fulfill communicative goals, we hypothesize that the designer chooses a design that best facilitates the perceptual and cognitive tasks that are most important to conveying his intended message, subject to the constraints imposed by competing tasks. By perceptual tasks we mean tasks that can be performed by simply viewing the graphic, such as finding the top of a bar in a bar chart; by cognitive tasks we mean tasks that are done via mental computations, such as computing the difference between two numbers.

Thus one source of evidence about the intended message is the relative difficulty of the perceptual tasks that the viewer would need to perform in order to recognize the message. For example, determining 
the entity with maximum value in a bar chart will be easiest if the bars are arranged in ascending or descending order of height. We have constructed a set of rules, based on research by cognitive psychologists, that estimate the relative difficulty of performing different perceptual tasks; these rules have been validated by eye-tracking experiments and are presented in (Elzer et al., 2004).

Another source of evidence is entities that have been made salient in the graphic by some kind of focusing device, such as coloring some elements of the graphic, annotations such as an asterisk, or an arrow pointing to a particular location in a graphic. Entities that have been made salient suggest particular instantiations of perceptual tasks that the viewer is expected to perform, such as comparing the heights of two highlighted bars in a bar chart.

And lastly, one would expect captions to help convey the intended message of an information graphic. The next section describes a corpus study that we performed in order to explore the usefulness of captions and how we might exploit evidence from them.

\section{A Corpus Study of Captions}

Although one might suggest relying almost exclusively on captions to interpret an information graphic, (Corio and Lapalme, 1999) found in a corpus study that captions are often very general. The objective of their corpus study was to categorize the kinds of information in captions so that their findings could be used in forming rules for generating graphics with captions.

Our project is instead concerned with recognizing the intended message of an information graphic. To investigate how captions might be used in a system for understanding information graphics, we performed a corpus study in which we analyzed the first 100 bar charts from our corpus of information graphics; this corpus contains a variety of bar charts from different publication venues. The following subsections present the results of this corpus study.

\subsection{Do Captions Convey the Intended Message?}

Our first investigation explored the extent to which captions capture the intended message of an information graphic. We extracted the first 100 graphics

\begin{tabular}{|l||r|}
\hline Category & $\#$ \\
\hline \hline Category-1: Captures intention (mostly) & 34 \\
Category-2: Captures intention (somewhat) & 15 \\
Category-3: Hints at intention & 7 \\
Category-4: No contribution to intention & 44 \\
\hline
\end{tabular}

Figure 3: Analysis of 100 Captions on Bar Charts

from our corpus of bar charts. The intended message of each bar chart had been previously annotated by two coders. The coders were asked to identify 1) the intended message of the graphic using a list of 12 high-level intentions (see Section 5 for examples) and 2) the instantiation of the parameters. For example, if the coder classified the intended message of a graphic as Change-trend, the coder was also asked to identify where the first trend began, its general slope (increasing, decreasing, or stable), where the change in trend occurred, the end of the second trend, and the slope of the second trend. If there was disagreement between the coders on either the intention or the instantiation of the parameters, we utilized consensus-based annotation (Ang et al., 2002), in which the coders discussed the graphic to try to come to an agreement. As observed by (Ang et al., 2002), this allowed us to include the "harder" or less obvious graphics in our study, thus lowering our expected system performance. We then examined the caption of each graphic, and determined to what extent the caption captured the graphic's intended message. Figure 3 shows the results. 44\% of the captions in our corpus did not convey to any extent the message of the information graphic. The following categorizes the purposes that these captions served, along with an example of each:

- general heading (8 captions): "UGI Monthly Gas Rates" on a graphic conveying a recent spike in home heating bills.

- reference to dependent axis (15 captions): "Lancaster rainfall totals for July" on a graphic conveying that July-02 was the driest of the previous decade.

- commentary relevant to graphic (4 captions): "Basic performers: One look at the best performing stocks in the Standard\&Poor's 500 index this year shows that companies with basic businesses are rewarding investors" on a 
graphic conveying the relative rank of different stocks, some of which were basic businesses and some of which were not. This type of information was classified as deductive by (Corio and Lapalme, 1999) since it draws a conclusion from the data depicted in the graphic.

- commentary extending message of graphic (8 captions): "Profits are getting squeezed" on a graphic conveying that Southwest Airlines net income is estimated to increase in 2003 after falling the preceding three years. Here the commentary does not draw a conclusion from the data in the graphic but instead supplements the graphic's message. However this type of caption would probably fall into the deductive class in (Corio and Lapalme, 1999).

- humor (7 captions): "The Sound of Sales" on a graphic conveying the changing trend (downward after years of increase) in record album sales. This caption has nothing to do with the change-trend message of the graphic, but appears to be an attempt at humor.

- conclusion unwarranted by graphic (2 captions): "Defense spending declines" on a graphic that in fact conveys that recent defense spending is increasing.

Slightly over half the captions (56\%) contributed to understanding the graphic's intended message. $34 \%$ were judged to convey most of the intended message. For example, the caption "Tennis players top nominees" appeared on a graphic whose intended message is to convey that more tennis players were nominated for the 2003 Laureus World Sports Award than athletes from any other sport. Since we argue that captions alone are insufficient for interpreting information graphics, in the few cases where it was unclear whether a caption should be placed in Category-1 or Category-2, we erred on the side of over-rating the contribution of a caption to the graphic's intended message. For example, consider the caption "Chirac is riding high in the polls" which appeared on a graphic conveying that there has been a steady increase in Chirac's approval ratings from $55 \%$ to about $75 \%$. Although this caption does not fully capture the communicative intention of the graphic (since it does not capture the steady increase conveyed by the graphic), we placed it in the first category since one might argue that riding high in the polls would suggest both high and improving ratings.

$15 \%$ of the captions were judged to convey only part of the graphic's intended message; an example is "Drug spending for young outpace seniors" that appears on a graphic whose intended message appears to be that there is a downward trend by age for increased drug spending; we classified the caption in Category-2 since the caption fails to capture that the graphic is talking about percent increases in drug spending, not absolute drug spending, and that the graphic conveys the downward trend for increases in drug spending by age group, not just that increases for the young were greater than for the elderly.

$7 \%$ of the captions were judged to only hint at the graphic's message. An example is "GM's Money Machine" which appeared on a graphic whose intended message was a contrast of recent performance against the previous trend - ie., that although there had been a steady decrease in the percentage of GM's overall income produced by its finance unit, there was now a substantial increase in the percentage provided by the finance unit. Since the term money machine is a colloquialism that suggests making a lot of money, the caption was judged to hint at the graphic's intended message.

\subsection{Understanding Captions}

For the 49 captions in Category 1 or 2 (where the caption conveyed at least some of the message of the graphic), we examined how well the caption could be parsed and understood by a natural language system. We found that $47 \%$ were fragments (for example, "A Growing Biotech Market"), or involved some other kind of ill-formedness (for example, "Running tops in sneaker wear in 2002" or "More seek financial aid"1). 16\% would require extensive domain knowledge or analogical reasoning to understand. One example is "Chirac is riding high in the polls" which would require understanding the meaning of riding high in the polls. Another example is "Bad Moon Rising"; here the verb rising suggests that something is increasing, but the

\footnotetext{
${ }^{1}$ Here we judge the caption to be ill-formed due to the ellipsis since More should be More students.
} 
system would need to understand that a bad moon refers to something undesirable (in this case, delinquent loans).

\subsection{Simple Evidence from Captions}

Although our corpus analysis showed that captions can be helpful in understanding the message conveyed by an information graphic, it also showed that full understanding of a caption would be problematic; moreover, once the caption was understood, we would still need to relate it to the information extracted from the graphic itself, which appears to be a difficult problem.

Thus we began investigating whether shallow processing of the caption might provide evidence that could be effectively combined with other evidence obtained from the graphic itself. Our analysis provided the following observations:

- Verbs in a caption often suggest the kind of message being conveyed by the graphic. An example from our corpus is "Boating deaths decline"; the verb decline suggests that the graphic conveys a decreasing trend. Another example from our corpus is "American Express total billings still lag"; the verb lag suggests that the graphic conveys that some entity (in this case American Express) is ranked behind some others.

- Adjectives in a caption also often suggest the kind of message being conveyed by the graphic. An example from our corpus is "Air Force has largest percentage of women"; the adjective largest suggests that the graphic is conveying an entity whose value is largest. Adjectives derived from verbs function similarly to verbs. An example from our corpus is "Soaring Demand for Servers" which is the caption on a graphic that conveys the rapid increase in demand for servers. Here the adjective soaring is derived from the verb soar, and suggests that the graphic is conveying a strong increase.

- Nouns in a caption often refer to an entity that is a label on the independent axis. When this occurs, the caption brings the entity into focus and suggests that it is part of the intended message of the graphic. An example from our cor- pus is "Germans miss their marks" where the graphic displays a bar chart that is intended to convey that Germans are the least happy with the Euro. Words that usually appear as verbs, but are used in the caption as a noun, may function similarly to verbs. An example is "Cable On The Rise"; in this caption, rise is used as a noun, but suggests that the graphic is conveying an increase.

\section{Utilizing Evidence}

We developed and implemented a probabilistic framework for utilizing evidence from a graphic and its caption to hypothesize the graphic's intended message. To identify the intended message of a new information graphic, the graphic is first given to a Visual Extraction Module (Chester and Elzer, 2005) that is responsible for recognizing the individual components of a graphic, identifying the relationship of the components to one another and to the graphic as a whole, and classifying the graphic as to type (bar chart, line graph, etc.); the result is an XML file that describes the graphic and all of its components.

Next a Caption Processing Module analyzes the caption. To utilize verb-related evidence from captions, we identified a set of verbs that would indicate each category of high-level goal ${ }^{2}$, such as recover for Change-trend and beats for Relative-difference; we then extended the set of verbs by examining WordNet for verbs that were closely related in meaning, and constructed a verb class for each set of closely related verbs. Adjectives such as more and most were handled in a similar manner. The Caption Processing Module applies a part-of-speech tagger and a stemmer to the caption in order to identify nouns, adjectives, and the root form of verbs and adjectives derived from verbs. The XML representation of the graphic is augmented to indicate any independent axis labels that match nouns in the caption, and the presence of a verb or adjective class in the caption.

The Intention Recognition Module then analyzes the XML file to build the appropriate Bayesian network; the current system is limited to bar charts, but

\footnotetext{
${ }^{2}$ As described in the next paragraph, there are 12 categories of high-level goals.
} 
the principles underlying the system should be extendible to other kinds of information graphics. The network is described in (Elzer et al., 2005). Very briefly, our analysis of simple bar charts has shown that the intended message can be classified into one of 12 high-level goals; examples of such goals include:

- Change-trend: Viewer to believe that there is a <slope-1> trend from <paraml> to $<$ param $2>$ and a significantly different $<$ slope-2> trend from $<$ param $3>$ to $<$ param $4>$

- Relative-difference: Viewer to believe that the value of element $<$ paraml $>$ is $<$ comparison $>$ the value of element <param $2>$ where $<$ comparison $>$ is greater-than, less-than, or equal-to.

Each category of high-level goal is represented by a node in the network (whose parent is the top-level goal node), and instances of these goals (ie., goals with their parameters instantiated) appear as children with inhibitory links (Huber et al., 1994) capturing their mutual exclusivity. Each goal is broken down further into subtasks (perceptual or cognitive) that the viewer would need to perform in order to accomplish the goal of the parent node. The network is built dynamically when the system is presented with a new information graphic, so that nodes are added to the network only as suggested by the graphic. For example, low-level nodes are added for the easiest primitive perceptual tasks and for perceptual tasks in which a parameter is instantiated with a salient entity (such as an entity colored differently from others in the graphic or an entity that appears as a noun in the caption), since the graphic designer might have intended the viewer to perform these tasks; then higher-level goals that involve these tasks are added, until eventually a link is established to the top-level goal node.

Next evidence nodes are added to the network to capture the kinds of evidence noted in Sections 3 and 4.3. For example, evidence nodes are added to the network as children of each low-level perceptual task; these evidence nodes capture the relative difficulty (categorized as easy, medium, hard, or impossible) of performing the perceptual task as esti- mated by our effort estimation rules mentioned in Section 3, whether a parameter in the task refers to an entity that is salient in the graphic, and whether a parameter in the task refers to an entity that is a noun in the caption. An evidence node, indicating for each verb class whether that verb class appears in the caption (either as a verb, or as an adjective derived from a verb, or as a noun that can also serve as a verb) is added as a child of the top level goal node. Adjectives such as more and most that provide evidence are handled in a similar manner.

In a Bayesian network, conditional probability tables capture the conditional probability of a child node given the value of its parent(s). For example, the network requires the conditional probability of an entity appearing as a noun in the caption given that recognizing the intended message entails performing a particular perceptual task involving that entity. Similarly, the network requires the conditional probability, for each class of verb, that the verb class appears in the caption given that the intended message falls into a particular intention category. These probabilities are learned from our corpus of graphics, as described in (Elzer et al., 2005).

\section{Evaluation}

In this paper, we are particularly interested in whether shallow processing of captions can contribute to recognizing the intended message of an information graphic. As mentioned earlier, the intended message of each information graphic in our corpus of bar charts had been previously annotated by two coders. To evaluate our approach, we used leave-one-out cross validation. We performed a series of experiments in which each graphic in the corpus is selected once as the test graphic, the probability tables in the Bayesian network are learned from the remaining graphics, and the test graphic is presented to the system as a test case. The system was judged to fail if either its top-rated hypothesis did not match the intended message that was assigned to the graphic by the coders or the probability rating of the system's top-rated hypothesis did not exceed $50 \%$. Overall success was then computed by averaging together the results of the whole series of experiments.

Each experiment consisted of two parts, one in 


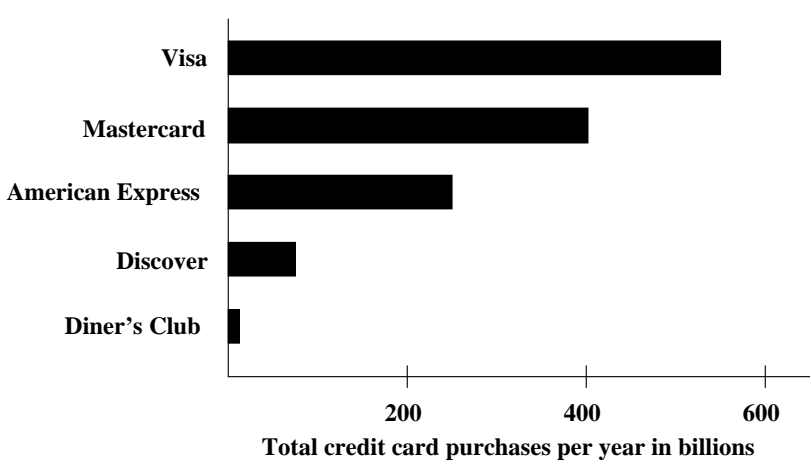

Figure 4: A Graphic from Business Week ${ }^{3}$

which captions were not taken into account in the Bayesian network and one in which the Bayesian network included evidence from captions. Our overall accuracy without the caption evidence was $64.5 \%$, while the inclusion of caption evidence increased accuracy to $79.1 \%$ for an absolute increase in accuracy of $14.6 \%$ and a relative improvement of $22.6 \%$ over the system's accuracy without caption evidence. Thus we conclude that shallow processing of a caption provides evidence that can be effectively utilized in a Bayesian network to recognize the intended message of an information graphic.

Our analysis of the results provides some interesting insights on the role of elements of the caption. There appear to be two primary functions of verbs. The first is to reflect what is in the data, thereby strengthening the message that would be recognized without the caption. One example from our corpus is a graphic with the caption "Legal immigration to the U.S. has been rising for decades". Although the early part of the graphic displays a change from decreasing immigration to a steadily increasing immigration trend, most of the graphic focuses on the decades of increasing immigration and the caption strengthens increasing trend in immigration as the intended message of the graphic. If we do not include the caption, our system hypothesizes an increasing trend message with a probability of $66.4 \%$; other hypotheses include an intended message that emphasizes the change in trend with a probability of $15.3 \%$. However, when the verb increasing from the caption is taken into account, the probability of increasing trend in immigration being the intended message rises to $97.9 \%$.

${ }^{3}$ This is a slight variation of the graphic from Business Week. In the Business Week graphic, the labels sometimes ap-
The second function of a verb is to focus attention on some aspect of the data. For example, consider the graphic in Figure 4. Without a caption, our system hypothesizes that the graphic is intended to convey the relative rank in billings of different credit card issuers and assigns it a probability of $72.7 \%$. Other possibilities have some probability assigned to them. For example, the intention of conveying that Visa has the highest billings is assigned a probability of $26 \%$. Suppose that the graphic had a caption of "Billings still lag"; if the verb lag is taken into account, our system hypothesizes an intended message of conveying the credit card issuer whose billings are lowest, namely Diner's Club; the probability assigned to this intention is now $88.4 \%$, and the probability assigned to the intention of conveying the relative rank of different credit card issuers drops to $7.8 \%$. This is because the verb class containing lag appeared in our corpus as part of the caption for graphics whose message conveyed an entity with a minimum value, and not with graphics whose message conveyed the relative rank of all the depicted entities. On the other hand, if the caption is "American Express total billings still lag" (which is the caption associated with the graphic in our corpus), then we have two pieces of evidence from the caption - the verb lag, and the noun American Express which matches a label. In this case, the probabilities change dramatically; the hypothesis that the graphic is intended to convey the rank of American Express (namely third behind Visa and Mastercard) is assigned a probability of $76 \%$ and the probability drops to $24 \%$ that the graphic is intended to convey that Diner's Club has the lowest billings. This is not surprising. The presence of the noun American Express in the caption makes that entity salient and is very strong evidence that the intended message places an emphasis on American Express, thus significantly affecting the probabilities of the different hypotheses. On the other hand, the verb class containing lag occurred both in the caption of graphics whose message was judged to convey the entity with the minimum value and in the caption of graphics

pear on the bars and sometimes next to them, and the heading for the dependent axis appears in the empty white space of the graphic instead of below the values on the horizontal axis as we show it. Our vision system does not yet have heuristics for recognizing non-standard placement of labels and axis headings. 
that conveyed an entity ranked behind some others. Therefore, conveying the entity with minimum value is still assigned a non-negligible probability.

\section{Future Work}

It is rare that a caption contains more than one verb class; when it does happen, our current system by default uses the first one that appears. We need to examine how to handle the occurrence of multiple verb classes in a caption. Occasionally, labels in the graphic appear differently in the caption. An example is DJIA (for Dow Jones Industrial Average) that occurs in one graphic as a label but appears as Dow in the caption. We need to investigate resolving such coreferences.

We currently limit ourselves to recognizing what appears to be the primary communicative intention of an information graphic; in the future we will also consider secondary intentions. We will also extend our work to other kinds of information graphics such as line graphs and pie charts, and to complex graphics, such as grouped and composite bar charts.

\section{Summary}

To our knowledge, our project is the first to investigate the problem of understanding the intended message of an information graphic. This paper has focused on the communicative evidence present in an information graphic and how it can be used in a probabilistic framework to reason about the graphic's intended message. The paper has given particular attention to evidence provided by the graphic's caption. Our corpus study showed that about half of all captions contain some evidence that contributes to understanding the graphic's message, but that fully understanding captions is a difficult problem. We presented a strategy for extracting evidence from a shallow analysis of the caption and utilizing it, along with communicative signals from the graphic itself, in a Bayesian network that hypothesizes the intended message of an information graphic, and our results demonstrate the effectiveness of our methodology. Our research is part of a larger project aimed at providing alternative access to information graphics for individuals with sight impairments.

\section{References}

J. Ang, R. Dhillon, A. Krupski, E. Shriberg, and A. Stolcke. 2002. Prosody-based automatic detection of annoyance and frustration in human-computer dialog. In Proc. of the Int'l Conf. on Spoken Language Processing (ICSLP).

D. Chester and S. Elzer. 2005. Getting computers to see information graphics so users do not have to. To appear in Proc. of the 15th Int'l Symposium on Methodologies for Intelligent Systems.

H. Clark. 1996. Using Language. Cambridge University Press.

M. Corio and G. Lapalme. 1999. Generation of texts for information graphics. In Proc. of the 7th European Workshop on Natural Language Generation, 49-58.

S. Elzer, S. Carberry, N. Green, and J. Hoffman. 2004. Incorporating perceptual task effort into the recognition of intention in information graphics. In Proceedings of the 3rd Int'l Conference on Diagrams, LNAI 2980, 255-270.

S. Elzer, S. Carberry, I. Zukerman, D. Chester, N. Green, S. Demir. 2005. A probabilistic framework for recognizing intention in information graphics. To appear in Proceedings of the Int'l Joint Conf. on AI (IJCAI).

R. Futrelle and N. Nikolakis. 1995. Efficient analysis of complex diagrams using constraint-based parsing. In Proc. of the Third International Conference on Document Analysis and Recognition.

R. Futrelle. 1999. Summarization of diagrams in documents. In I. Mani and M. Maybury, editors, Advances in Automated Text Summarization. MIT Press.

Nancy Green, Giuseppe Carenini, Stephan Kerpedjiev, Joe Mattis, Johanna Moore, and Steven Roth. Autobrief: an experimental system for the automatic generation of briefings in integrated text and information graphics. International Journal of Human-Computer Studies, 61(1):32-70, 2004.

H. P. Grice. 1969. Utterer's Meaning and Intentions. Philosophical Review, 68:147-177.

M. Huber, E. Durfee, and M. Wellman. 1994. The automated mapping of plans for plan recognition. In Proc. of Uncertainty in AI, 344-351.

S. Kerpedjiev and S. Roth. 2000. Mapping communicative goals into conceptual tasks to generate graphics in discourse. In Proc. of Int. Conf. on Intelligent User Interfaces, 60-67.

J. Yu, J. Hunter, E. Reiter, and S. Sripada. 2002. Recognising visual patterns to communicate gas turbine time-series data. In ES2002, 105-118. 